\title{
Glucose injections into the dorsal hippocampus or dorsolateral striatum of rats prior to T-maze training: Modulation of learning rates and strategy selection
}

\author{
Clinton E. Canal, ${ }^{1}$ Sonja J. Stutz, ${ }^{2}$ and Paul E. Gold ${ }^{1,2,3,4}$ \\ ${ }^{1}$ The Neuroscience Program, ${ }^{2}$ Department of Psychology and ${ }^{3}$ Department of Psychiatry, University of Illinois, \\ Urbana-Champaign, Illinois 61820, USA
}

\begin{abstract}
The present experiments examined the effects of injecting glucose into the dorsal hippocampus or dorsolateral striatum on learning rates and on strategy selection in rats trained on a T-maze that can be solved by using either a hippocampus-sensitive place or striatum-sensitive response strategy. Percentage strategy selection on a probe trial $\left(\mathrm{P}_{\text {crit }}\right)$ administered after rats achieved criterion (nine of 10 correct choices) varied by group. All groups predominately exhibited a response strategy on a probe trial administered after overtraining, i.e., after 90 trials. In experiment 1, rats that received intrahippocampal glucose injections showed enhanced acquisition of the T-maze and showed increased use of response solutions at $P_{\text {crit }}$ compared with that of unimplanted and artificial cerebral spinal fluid (aCSF)-treated groups. These findings suggest that glucose enhanced hippocampal functions to accelerate the rate of learning and the early adoption of a response strategy. In experiment 2, rats that received intrastriatal glucose injections exhibited place solutions early in training and reached criterion more slowly than did aCSF controls, with learning rates comparable to those of unoperated and operated-uninjected controls. Relative to unoperated, operated-uninjected and glucose-injected rats, rats that received intrastriatal aCSF injections showed enhanced acquisition of the T-maze and increased use of response solutions at $P_{\text {crit }}$. The unexpected enhanced acquisition seen after striatal aCSF injections suggests at least two possible interpretations: (1) aCSF impaired striatal function, thereby releasing competition with the hippocampus and ceding control over learning to the hippocampus during early training trials; and (2) aCSF enhanced striatal functioning to facilitate striatal-sensitive learning. With either interpretation, the results indicate that intrastriatal glucose injections compensated for the aCSF-induced effect. Finally, enhanced acquisition regardless of treatment was accompanied by rapid adoption of a response solution for the T-maze.
\end{abstract}

Glucose is a potent modulator of learning and memory for many tasks in both humans and rodents (Gold 1995, 2001; Korol and Gold 1998). For example, systemic administration of glucose enhances memory for verbal tasks in humans (cf. Gold 2001; Korol 2002; Benton et al. 2003; Messier 2004; Watson and Craft 2004) and enhances learning and memory in rats and mice for appetitive, aversive, operant, visual discrimination, spatial working memory, habituation, and extinction tasks (cf. Messier and White 1984, 1987; Gold 1986, 1995; Messier and Destrade 1988; Kopf and Baratti 1996; Messier 1997; Pavone et al. 1998; Ragozzino et al. 1998; Sansone et al. 2000; Talley et al. 2000; Benton et al. 2003; Hughes 2003; Schroeder and Packard 2003).

In addition to the effects seen with systemic injections, glucose can also enhance learning and memory when injected directly into specific brain regions. Glucose injections into the medial septum or hippocampus modulate memory of spontaneous alternation tasks (see Ragozzino et al. 1992, 1998; Parent and Gold 1997; Parent et al. 1997; Stefani and Gold, 1998, 2001; Degroot et al. 2003; Shah and Parent 2003). In addition, intraamygdala glucose injections enhance consolidation of extinction of drug-induced conditioned reward (Schroeder and Packard

\footnotetext{
${ }^{4}$ Corresponding author.

E-mail pgold@uiuc.edu; fax (217) 244-5876.

Article published online ahead of print. Article and publication date are at http://www.learnmem.org/cgi/doi/10.1101/lm.88205.
}

2003) and reverse memory impairments for inhibitory avoidance training caused by intra-amygdala morphine (Ragozzino and Gold 1994) and deficits on a spontaneous alternation task caused by intraseptal morphine (Ragozzino and Gold 1995; McNay and Gold 1998). Despite these findings, the evidence for enhancement of learning and memory with direct central injections of glucose remains sparse. In addition, there are no prior attempts to integrate glucose effects on memory with considerations of interactions between memory systems important for processing information for different tasks; examinations of these interactions was a primary goal of the present experiments.

Considerable evidence supports the view that the hippocampus and striatum are important for processing information for different types of learning and memory (cf. Packard and Knowlton 2002; White and McDonald 2002; Gold 2004; Korol et al. 2004; Mizumori et al. 2004). Much of the support for this view comes from studies showing impairments after lesions of the hippocampal formation on tasks involving spatial, contextual, or win-shift learning but not on tasks involving cued, procedural, or win-stay learning. Lesions of the striatum produce the converse set of impairments (see Cook and Kesner 1988; Packard et al. 1989; Packard and McGaugh 1992; Kesner et al. 1993; McDonald and White 1993; Knowlton et al. 1996; Eichenbaum and Cohen 2001).

In addition to the patterns of impairments in learning observed after lesions, several studies have reported selective en- 
hancement of particular types of learning and memory following pharmacological manipulations of the hippocampus or striatum. Post-training amphetamine enhances later memory for a winstay task, but not a win-shift task, when injected into the striatum but enhances later memory for a win-shift task, and not a win-stay task, when injected into the hippocampus (Packard and White 1991). Similarly, post-training injections of amphetamine into the striatum facilitate later memory for training on a cued but not a spatial version of the water task. Conversely, injections into the hippocampus facilitate later retention for a spatial but not a cued version of the task (Packard et al. 1994). Thus, the findings of both lesion and pharmacological studies provide substantial support for the view that the hippocampus and striatum are neural systems that process information for different types of learning and memory.

Recent findings suggest that the interactions between the hippocampus and striatum might be characterized as competitive in nature (cf. Packard and Knowlton 2002; White and McDonald 2002; Poldrack and Packard 2003; Gold 2004; however, see Voermans et al. 2004). In particular, interference with the functioning of one neural system can facilitate behavioral expression of memory sensitive to interference with the functioning of another system. For example, lesions of the striatum enhance acquisition of a spatial Y-maze discrimination task, a task impaired by lesions of the hippocampus (Mitchell and Hall 1988). Conversely, lesions of the fimbria/fornix, often shown to impair spatial learning (see Olton et al. 1979; Walker and Olton 1979; Cassel et al. 1998), enhance nonspatial learning in rats (Matthews et al. 1999). Findings supporting the notion of competitive interactions between the hippocampus and striatum have also been evident with pharmacological manipulations. Inactivation of the hippocampus with infusions of lidocaine prior to training impairs acquisition of a spatial version of the plus-maze task yet enhances acquisition of a response version of the task (Chang and Gold 2003b). Similarly, post-training intrahippocampal infusions of bupivacaine attenuate acquisition of a spatial version of a water plus-maze task and enhance acquisition of a response version of the task (Schroeder et al. 2002). These results are consistent with the hypothesis that one neural system (e.g., hippocampus) may process information that is not useful when solving a task that is predominantly dependent on another system (e.g., striatum), thereby interfering with learning of the task (cf. White and McDonald 2002; Poldrack and Packard 2003; Gold 2004).

The differences in participation of the hippocampus and striatum to different types of learning and memory imply that pharmacological agents that enhance memory when injected into the hippocampus or striatum might influence the strategy a rat prefers when learning a task that can be solved successfully with either of two strategies. The T-maze (Tolman et al. 1946, 1947; Restle 1957) can be solved by using either a hippocampussensitive place or a striatum-sensitive response strategy (Packard and McGaugh 1996; Packard 1999). When rats are trained on this task, they generally use a place solution early in training and then switch to expressing a response solution later in training, suggesting that hippocampal processes control expression of learning in early training trials but that striatal processes take over later in training (Packard and McGaugh 1996; Packard 1999; Chang and Gold 2003a). However, this behavioral phenomenon depends on intra- and extramaze cue arrangements and densities (Tolman et al. 1946, 1947).

Post-training injections of glutamate into the hippocampus prolong the use of a place strategy and block the switch to a response strategy when rats are trained across days in a T-maze. Conversely, post-training glutamate injections into the striatum result in rats using response strategies early in training as well as late in training. These findings suggest that manipulations of neurotransmitters within a particular neural system modulate the relative participation of that structure during learning and memory processing (Packard 1999). Also consistent with this view, the pattern of acetylcholine (ACh) release in the hippocampus and striatum during training is associated with the use of place and response solutions (Chang and Gold 2003a; McIntyre et al. 2003; cf. Gold 2003). Importantly, both neurochemical and physiological findings suggest that while there may be competition in terms of the learned responses expressed when memory is tested, both the hippocampus and striatum are engaged during learning in the T-maze and are important processors for T-maze learning (White and McDonald 2002; Chang and Gold 2003a; Mizumori et al. 2004).

The primary question we investigated in the present experiments was whether pre-training injections of glucose into either the dorsal hippocampus or dorsolateral striatum would modulate learning and strategy selection during massed training in a Tmaze.

\section{Results}

\section{Experiment 1: Effects of intrahippocampal injections of glucose on T-maze learning}

Early learning rates (trial blocks 1-3) in the glucose-treated group were significantly faster than those in the artificial cerebral spinal fluid (aCSF) group $\left.\left[F_{(1,30)}=4.74 ; P<0.05\right)\right]$ (Fig. 1$)$. As shown in Figure 2 , rats that received glucose injections reached criterion in fewer trials than did rats that received hippocampal aCSF injections [mean $( \pm$ SEM) $=21(2.4), 30$ (3.9), respectively; $P<0.05$, or rats that served as unoperated controls [29 (2.5); $P<0.05]$. The number of trials to criterion was comparable in rats that received dorsal hippocampus aCSF injections and unoperated rats $(P \geq 0.8)$.

Figure 3 shows the relative strategy selection on the first probe trial administered after criterion of nine of 10 correct $\left(\mathrm{P}_{\text {crit }}\right)$ used by the unoperated group and by groups receiving intrahippocampal injections of aCSF or glucose. Unoperated control rats expressed equivalent use of a place or response strategy at $\mathrm{P}_{\text {crit }}$ (50\% place vs. $50 \%$ response). Similarly, rats that received dorsal hippocampus aCSF injections showed similar use of a place or response strategy at $\mathrm{P}_{\text {crit }}$ (53\% place vs. $47 \%$ response). In contrast, rats that received glucose injections into the dorsal hippocampus tended to exhibit a response solution at $\mathrm{P}_{\text {crit }}(29 \%$ place vs. $71 \%$ response; $P<0.1 ; \chi^{2}=2.88$ ). On the final probe trial administered after completion of 90 training trials, all groups exhibited predominately response solutions (58\%-80\% response; data not shown).

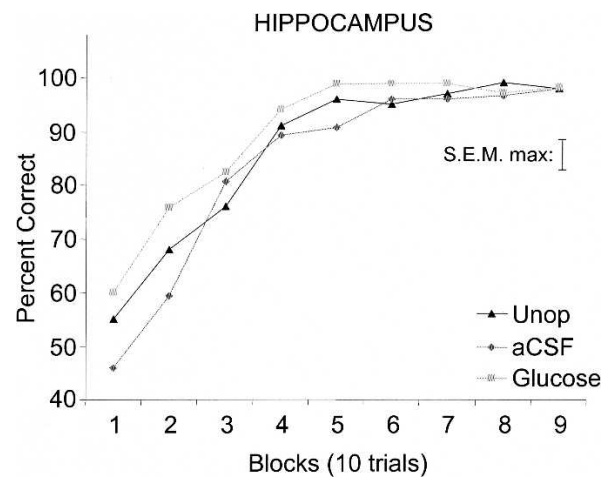

Figure 1. Learning curves for groups receiving glucose or aCSF injections into dorsal hippocampus and for unoperated (Unop) controls. The hippocampus glucose group exhibited more rapid learning (blocks 1-3) than did the aCSF group $(P<0.05)$. 


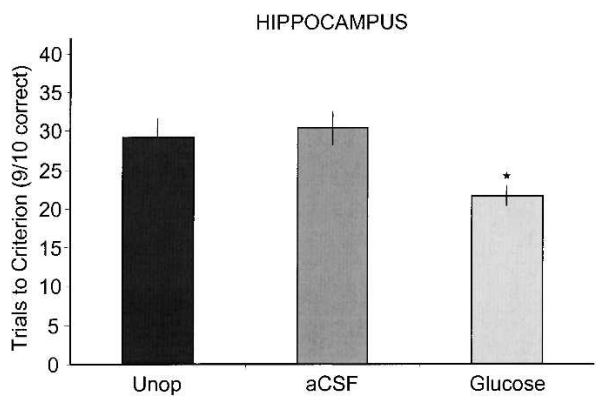

Figure 2. Trials to criterion for groups receiving glucose or aCSF injections into the dorsal hippocampus and for unoperated controls. The group that received pretraining injections of glucose into the hippocampus reached criterion (nine of 10 correct) in a T-maze in significantly fewer trials than did either the aCSF or unoperated (Unop) groups. Asterisk indicates $P<0.05$.

\section{Discussion}

Intrahippocampal injections of glucose administered shortly before training enhanced the rate of acquisition of a T-maze. Glucose also modulated the pattern of place versus response solutions selected by the rats on the probe trial administered soon after each rat reached criterion.

The enhancement in learning rates by glucose is consistent with enhancement by intrahippocampal glucose of memory scores on a spontaneous alternation task (Ragozzino et al. 1998; Stefani and Gold 2001). While the present findings, to our knowledge, represent the first demonstration of enhancement of appetitive maze learning by infusions of glucose into the hippocampus, the findings are consistent with evidence obtained with other treatments showing that intrahippocampal injections of drugs that generally activate the hippocampus enhance learning and memory for several tasks. For example, intrahippocampal injections of corticosterone or cysteamine enhance appetitive operant conditioning (Micheau et al. 1984; Guillou et al. 1999). In addition, intrahippocampal injections of amphetamine, bicuculline, nicotine, or corticosterone enhance inhibitory avoidance memory (Lee and Ma 1995; Roozendaal et al. 1999; Luft et al. 2004; Marta-Barros et al. 2004), and estrogen injections enhance memory for a spatial swim task (Packard et al. 1996; Packard 1998) and for an appetitive place task (Korol 2004).

Particularly relevant here are the results obtained with a test of the effects of intrahippocampal glutamate injections on Tmaze learning (Packard 1999). In contrast to the glucose enhancement of the rate of acquisition and expression of a response strategy seen here, glutamate had no evident effects on learning rates, but robustly enhanced and prolonged expression of a place strategy revealed on probe trials. A key difference between the present experiment and Packard (1999) is the use of different drug treatments. However, there are also other important differences in the methods employed, including pre-training versus post-training injections, and training administered within a single session versus training administered at four trials per day for $16 \mathrm{~d}$ in the present and Packard (1999) experiments, respectively. In the present experiments, pre-training intrahippocampal glucose injections enhanced the rate of learning and therefore may have enhanced an early switch to response solutions.

\section{Results}

Experiment 2: Effects of intrastriatal injections of glucose on T-maze learning

Rats that received aCSF injections into the dorsolateral striatum exhibited more rapid learning than did cannulae-implanted rats that did not receive injections (no injection) or unoperated rats. The rats that received injections of glucose into the dorsolateral striatum had learning curves comparable to those of no injection and unoperated rats and slower than those of aCSF-treated rats. As shown in Figure 4, the initial rates of learning revealed that the aCSF group showed significantly enhanced learning rates compared to those of the glucose $\left[F_{(1,14)}=7.95 ; P<0.01\right]$, no injection $\left[F_{(1,11)}=19.89 ; P<0.01\right]$, or unoperated $\left[F_{(1,15)}=10.36\right.$; $P<0.01]$ groups. The rapid learning in the aCSF group compared with either glucose, no injection, or unoperated controls groups was also revealed in analyses of trials to criterion (Fig. 5). The rats that received dorsolateral striatum aCSF injections reached criterion performance more quickly than did glucose $(P<0.01)$, no injection $(P<0.01)$, and unoperated $(P<0.01)$ rats. In addition, rats administered glucose reached criterion more slowly than did unoperated rats $(P<0.07)$.

As shown in Figure 6, rats that received aCSF injections into the dorsolateral striatum tended to use a response solution at $\mathrm{P}_{\text {crit }}$ (29\% place vs. $71 \%$ response). The glucose and no injection groups showed a preference for the place solution at $\mathrm{P}_{\text {crit }}[78 \%$ place, $22 \%$ response $\left(\chi^{2}=2.76, P<0.1\right), 67,33 \%$, respectively]. Thus, the aCSF group learned most rapidly and showed early use of a response solution. In contrast, the glucose and no injection groups learned more slowly and showed early use of a place solution. On the final probe trial administered after completion of 90 training trials, all groups exhibited predominately response solutions (57\%-70\% response; data not shown).

\section{Discussion}

The interpretation of the effects of injections of glucose into the dorsolateral striatum on learning and on strategy selection during T-maze training is complicated by the difference in learning rates exhibited in the aCSF-injected group. One interpretation of these findings is that, early in training, the hippocampus and striatum compete for control over learning. According to this view, aCSF injections may have impaired striatal function, leading to augmented hippocampal control over early learning, a stage of learning in the T-maze that is putatively mediated by the hippocampus (cf. Packard and McGaugh 1996). This interpretation is consistent with similar findings after striatal damage obtained in a different task (Mitchell and Hall 1988) and fits into a broader set of findings showing enhanced learning and memory on specific tasks after selective interference with the functions of some neural systems. For example, enhanced memory for nonspatial learning and for conditioned cue preference training are evident after damage to the hippocampal formation (White and McDonald 1993; McDonald and White 1995; Matthews et al. 1999; Ferbinteanu and McDonald 2001). Furthermore, using re-

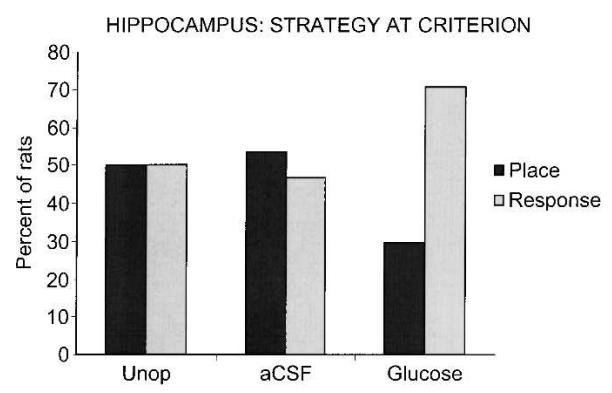

Figure 3. Use of place vs. response solutions on the first probe trial after each rat reached criterion of nine of 10 correct, $P_{\text {crit }}$ (hippocampus). Note that the hippocampus glucose group, in which learning was most rapid, predominantly exhibited a response solution. 


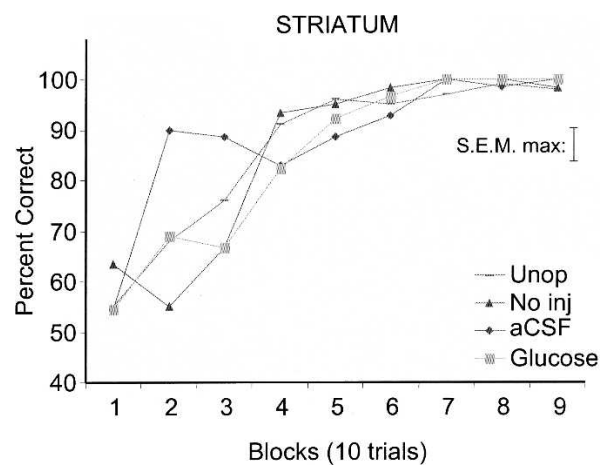

Figure 4. Learning curves for groups receiving glucose, aCSF or no injections (No inj) into dorsolateral striatum. Note that the group that received pre-training aCSF injections into the striatum exhibited more rapid learning than did either the No inj or Glucose groups ( $P_{s} \leq 0.01$ ).

lease of ACh in the hippocampus as a measure of the extent of engagement the hippocampus has during training, the magnitude of release of ACh in the hippocampus is inversely related to acquisition of a conditioned cue preference task, a task sensitive to amygdala lesions and to direct pharmacological manipulations (McIntyre et al. 2002). An alternative interpretation is that striatal aCSF injections enhanced striatal function, thereby enhancing striatal-sensitive learning (e.g. response learning) and facilitating a response strategy. While the nature of the functional interactions between hippocampus and striatum is not well understood, functional interactions between these brain systems are also seen in humans in young adults (Poldrack et al. 2001; Iaria et al. 2003; cf: Packard and Knowlton 2002; Poldrack and Packard 2003; Poldrack and Rodriguez 2004) and in comparisons of healthy subjects and individuals with Huntington's disease (Voermans et al. 2004).

The mechanistic effects of aCSF or glucose injections into the dorsolateral striatum are unclear. However, similar to the present findings, other studies have also shown reversal of learning and memory deficits by treatments administered at a site of damage. For example, rats exhibited deficits in memory for inhibitory avoidance training after implantation of electrodes into the mesencephalic reticular formation (Denti et al. 1970) or amygdala (Gold et al. 1978). In both cases, electrical stimulation through the electrodes at the time of training reversed the deficits, i.e., enhanced memory. The findings of the present experiment imply that the effects of aCSF injections into the dorsolateral striatum were reversed by glucose injections into the same brain region. This is, to our knowledge, the first instance in which a drug that generally enhances memory, i.e., glucose, apparently reinstated basal level interaction or competition between neural systems.

The results obtained on the probe trials are particularly interesting. Striatal glucose-treated rats exhibited slow rates of acquisition compared with that of the aCSF group and showed a strong tendency to use a place strategy early in training. In the rats in the striatal aCSF group, rapid acquisition was accompanied by a preference for a response solution. Thus, earlier adoption of a response solution appeared in those rats that showed rapid acquisition relative to those that showed slow acquisition in the T-maze. Confirming this observation, as shown in Figure 7 , combining the data from all rats in both experiments and separating rats by use of a response or place strategy at $\mathrm{P}_{\text {crit }}$ revealed that, regardless of group, rats that exhibited early use of a response strategy showed enhanced acquisition of the T-maze $(P<0.04)$.

\section{General discussion}

The T-maze is an attractive task with which to examine interactions between memory systems because a rat can successfully learn to obtain a food reward by using either place or response solutions, sensitive to hippocampus or striatum manipulations, respectively (Tolman et al. 1946, 1947; Restle 1957; Packard and McGaugh 1996; Packard 1999; Chang and Gold 2003a; McIntyre et al. 2003). Given this dissociation, it seemed plausible that acquisition rates would be unaltered by glucose infusions into these structures, but preferred solution strategies would differ. However, there were indeed clear differences in rates of acquisition across groups. Compared with the unoperated and the hippocampus aCSF groups (Experiment 1), acquisition of the T-maze was facilitated by intrahippocampal injections of glucose. However, in the dorsolateral striatum experiment (Experiment 2), the aCSF group exhibited accelerated learning compared with unoperated, no injection, and glucose-treated rats. Injections of glucose into the striatum slowed learning rates to those seen in no injection rats. Thus, contrary to the hypothesis that intrahippocampal or intrastriatal glucose would not change the rate of acquisition but would change only the strategy expressed on probe trials, glucose enhanced acquisition relative to aCSF controls when injected into the dorsal hippocampus and impaired acquisition relative to the aCSF controls when injected into the dorsolateral striatum.

A second hypothesis was that, as seen with glutamate injections (Packard 1999), injections of glucose into the hippocampus would result in preference for place solutions on probe trials, while injections of glucose into the striatum would result in response solutions. The results showed instead that injections of glucose into the dorsal hippocampus facilitated early use of a response strategy, while injections of glucose into the striatum facilitated early use of a place strategy. The preferred strategy expressed on the probe trial administered soon after the rats had met the criterion of nine of 10 correct, $\mathrm{P}_{\text {crit }}$, was related to the rate of acquisition. The groups that showed relatively slow rates of acquisition (hippocampus aCSF, striatum no injection, striatum glucose) all expressed a predominant place bias or no bias at $\mathrm{P}_{\text {crit }}$. In contrast, the groups that showed rapid acquisition (hippocampus glucose, striatum aCSF) predominantly expressed response solutions at $\mathrm{P}_{\text {crit }}$. In addition, this observation was evident when rats from all groups were separated according to strategy at $P_{\text {crit }}$. Thus, a preference for a place solution early in training was evident under conditions of relatively slower learning, while a preference for response solutions was evident early in training for those rats with accelerated rates of learning, results similar to those of McEl-

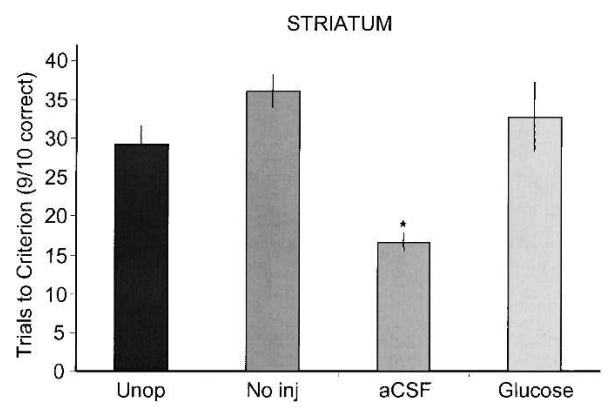

Figure 5. Trials to criterion for groups receiving glucose, aCSF, or no injections (No inj) into the dorsolateral striatum. The unoperated controls shown in Experiment 1 are included here for comparison. The group that received pre-training injections of aCSF into the striatum reached criterion (nine of 10 correct) in the T-maze in significantly fewer trials than did either the unoperated (Unop), No inj, or glucose groups. Asterisk indicates $P \leq 0.05$. 


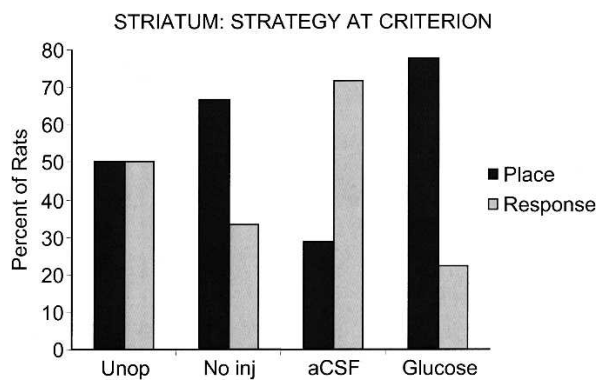

Figure 6. Use of place vs. response solutions on the first probe trial after each rat reached criterion of nine of 10 correct, $P_{\text {crit. }}$. Note that the striatal glucose and no injection (No inj) groups showed a place strategy preference. However, the aCSF group, in which learning was most rapid, predominantly exhibited a response strategy.

roy and Korol (2005). These findings suggest at least two possibilities: (1) accelerated learning is accompanied by accelerated transition from place to response solutions, resulting in such transitions occurring prior to the first probe tests in the present experiments; and (2) early selection of a response strategy leads to accelerated learning of the T-maze.

In assessing the release of ACh in hippocampus and striatum during learning, the relative increases in ACh release during training were related to the preference for place or response solutions (McIntyre et al. 2002). It is interesting to note that increases in release of ACh in the hippocampus preceded the increases of release of ACh in the striatum, suggesting that $\mathrm{ACh}$ is a marker of the transition from hippocampus-sensitive place solutions to striatum-sensitive response solutions in the T-maze (Chang and Gold 2003a). It will be important to determine whether dynamic responses of ACh release in the two neural systems reflect enhanced learning, as seen in this experiment.

The efficacy of infusions of glucose into the dorsal hippocampus and dorsolateral striatum in modulating learning and memory may be related to findings that dynamic changes in extracellular glucose levels in the brain are associated with memory processing (cf. McNay et al. 2000; Gold 2001; McNay and Gold 2002; McNay and Sherwin 2004). Of particular interest here, glucose levels in the hippocampus, but not striatum, decrease when rats are tested on a hippocampus-dependent spontaneous alternation task. Peripheral injections of glucose block the depletion of extracellular fluid glucose levels in the hippocampus while enhancing memory in a spontaneous alternation task (McNay et al. 2001). It is possible that direct glucose injections into both brain areas modulate learning and memory by blocking the depletion of glucose in the extracellular fluid. Yet to be conducted are tests of the possibility that extracellular glucose levels in the dorsolateral striatum are responsive to training on tasks sensitive to lesions of the dorsolateral striatum.

A mechanism by which central glucose modulates memory processes includes effects, likely indirect, on ACh release in a manner that appears to be related to memory processing. Systemic glucose injections increase hippocampal ACh release (Durkin et al. 1992), and both systemic and intrahippocampal glucose injections increase $\mathrm{ACh}$ release in the hippocampus while also enhancing spontaneous alternation performance (Ragozzino et al. 1996, 1998). Other putative memory-modulating mechanisms of glucose include regulation of potassium-ATP channels to modify neural excitability (Stefani and Gold 1998, 2001; Stefani et al. 1999; Rashidy-Pour 2001), and modulation of GABA neurotransmission (Degroot et al. 2003).

In summary, the findings of these experiments indicate that glucose injections into the dorsal hippocampus or dorsolateral striatum modulate learning in the T-maze and indicate that the generally observed progression of learning in the T-maze for untreated rats, from place to response, can be altered by manipulations of hippocampus or striatum function.

\section{Materials and Methods}

\section{Experiment 1}

\section{Subjects}

Male Sprague-Dawley rats (Harlan Laboratories), $\sim 3$ mo old, were individually housed and maintained on a 12-h light/12-h dark cycle (lights on at 0800) with free access to food and water for at least $1 \mathrm{wk}$ prior to surgery. All training was performed between 1200 and $1700 \mathrm{~h}$.

\section{Surgery}

Rats were anesthetized with sodium pentobarbital $(65 \mathrm{mg} / \mathrm{kg}$, i.p.), and treated with atropine sulfate $(0.27 \mathrm{mg} / \mathrm{kg}$, i.p.). By using standard stereotaxic procedures, sterile, stainless steel guide cannulae (22 gauge; Plastics One, Inc.) were implanted bilaterally into the dorsal hippocampus (nosebar, -3.3, AP, -4.0; $\mathrm{ML}$, \pm 2.9 ; DV,$-1.8 \mathrm{~mm}$ from dura) according to the atlas of Paxinos and Watson (1986). Saline $(0.9 \%$ solution, $6.0 \mathrm{~mL})$ was administered subcutaneously as supportive fluid following surgery, and buprenorphine $(0.05 \mathrm{mg} / \mathrm{kg}$, i.p.) was administered as postoperative analgesic.

\section{Training procedures}

Beginning $\geq 1$ wk after surgery, rats were placed on a food restriction schedule that reduced their body weights to $80 \%-85 \%$ of baseline and then maintained their weights at that level. Rats were handled daily, beginning at the start of food deprivation and continuing for at least $7 \mathrm{~d}$ until behavioral procedures were conducted. On each of the $3 \mathrm{~d}$ before testing, rats were given $~ 10$ Noyes pellets ( $45 \mathrm{mg}$ each) in their home cage to habituate them to the reward used during training.

Rats were trained on a four-arm, plus-shaped maze (arms: 45-cm length, 14-cm width, 7.5-cm height; center area: 14 $\mathrm{cm} \times 14 \mathrm{~cm}$ ) constructed of opaque, black Plexiglas. A removable Plexiglas barrier was used to block the entrance to one testing arm, rendering a T-shaped maze. The maze was placed in the center of the testing rooms on a table $76 \mathrm{~cm}$ above the floor and at least $65 \mathrm{~cm}$ away from a rich assortment of extramaze visual cues.

\section{Intracranial injections and behavioral testing}

aCSF (containing $0.5 \mathrm{nmol}$ glucose in $0.5 \mu \mathrm{L}$ ) or glucose $(16.7$ $\mathrm{nmol}$ in $0.5 \mu \mathrm{L}$ aCSF) was injected bilaterally into the dorsal hippocampus prior to training. The glucose dose used was shown previously to modulate memory with direct brain injections

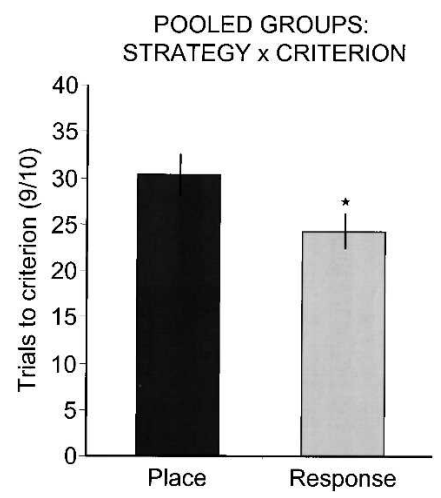

Figure 7. Strategy selection at $P_{\text {crit }}$ by trials to criterion across all groups. Rats that exhibited a response solution at $P_{\text {crit }}$ showed enhanced acquisition relative to rats that exhibited a place solution at $P_{\text {crit }}$. Asterisk indicates $P<0.05$. 


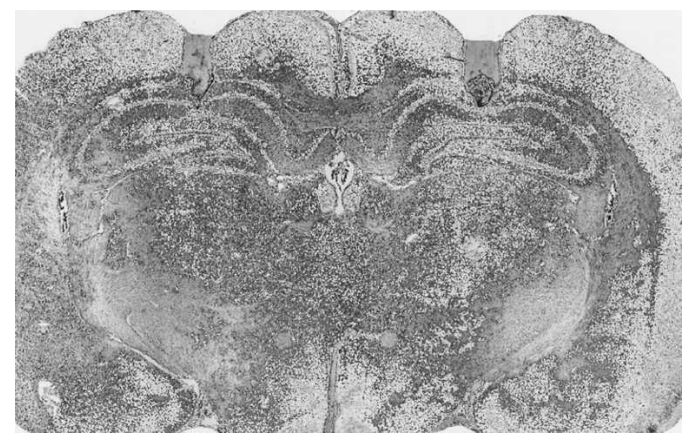

Figure 8. Photomicrograph of cannulae placements in the dorsal hippocampus.

(Ragozzino and Gold 1994; McNay and Gold 1998). A separate group of rats served as unoperated controls. Thus, the experimental design included three groups: unoperated $(\mathrm{N}=10)$, dorsal hippocampus aCSF $(\mathrm{N}=15)$, and dorsal hippocampus glucose $(\mathrm{N}=17)$.

Two 25- $\mu \mathrm{L}$ Hamilton syringes were each connected with polyethylene tubing to 28-gauge, sterile infusion cannulae. The cannulae protruded $1 \mathrm{~mm}$ beyond the guide cannulae pedestals when inserted. The syringes were attached to a CMA 100 pump (Carnegie Medicin). Infusion cannulae were gently inserted into the guide cannulae, and injections were made over a 2 -min period at a perfusion rate of $0.25 \mu \mathrm{L} / \mathrm{min}$. To allow diffusion of drug, the infusion cannulae were left in place $1 \mathrm{~min}$ following the injection.

Beginning $\sim 4$ min after the injection, each rat was placed in the east arm of the T-maze facing west and trained to approach either the south or north arm for two Noyes pellets. After making a choice, the rat was placed for $\sim 30 \mathrm{sec}$ in its home cage, located on the floor of the testing room. During this intertrial interval, the maze was rotated $90^{\circ}$ in a clockwise direction, using preset stops, to prevent the successful use of intramaze cues to reach the goal arm. After every 15 th training trial, the start arm of the maze was rotated $180^{\circ}$ relative to the training trials; rats were placed in the west arm facing east for a probe trial to assess strategy. During this probe trial, both arms were baited. Obtaining the reward by turning toward the spatial location of the reward was designated a "place" strategy. Obtaining the reward by turning in the same direction (left or right) as during training was termed a "response" strategy (Restle 1957). Rats received 90 training trials, with six probe trials. Percentage of correct choices, blocked into 10 consecutive trials, defined learning curves for each group. In addition, trials to criterion (nine of 10 correct) and strategy selection (place or response) were recorded. The results presented here are those obtained on the first probe trial after rats reached the nine of 10 criterion, designated $\mathrm{P}_{\text {crit }}$. Across all groups, $\mathrm{P}_{\text {crit }}$ occurred at a mean of $6.6 \pm 0.2$ SEM trials after rats reached the nine of 10 criterion. There were no significant differences be-

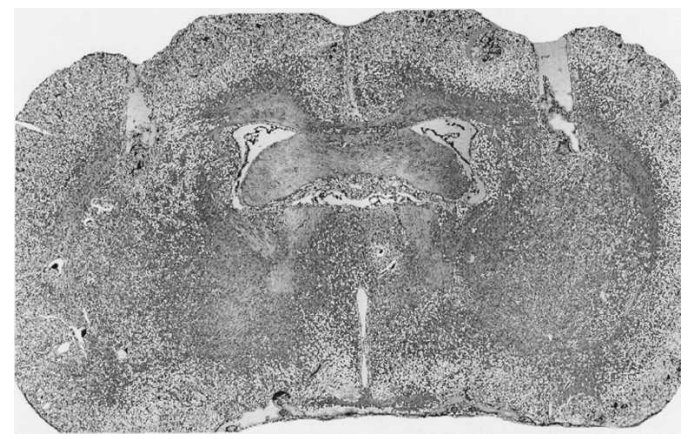

Figure 9. Photomicrograph of cannulae placements in the dorsolateral striatum. tween groups in the number of trials between reaching criterion and the next probe trial $(P s=0.66-0.97)$.

Because the hippocampus is particularly seizure-prone, electrographic records of the hippocampus were taken from additional rats before, during, and after injections of either aCSF $(\mathrm{N}=2)$ or glucose $(\mathrm{N}=2)$. No epileptiform or other gross electrographic abnormalities were observed (data not shown).

\section{Histology}

Following testing, subjects were euthanized by overdose with pentobarbital $(120 \mathrm{mg} / \mathrm{kg})$. Brains were removed and were stored in $10 \%$ formalin solution. The brains were later frozen and sectioned at $50 \mu \mathrm{m}$ on a Reichert-Jung cryostat. Sections were stained with cresyl violet, and placements of cannulae were identified. Figure 8 illustrates a representative example of cannulae placement in the dorsal hippocampus.

\section{Statistical analyses}

Paired $t$-tests were used to analyze differences in trials to criterion (nine of 10 correct) between glucose, aCSF, and unoperated groups. A single-factor, repeated-measures ANOVA test was used to analyze early learning differences (trial blocks $1-3$ ) between aCSF- and glucose-injected groups. $\chi^{2}$ tests were used to assess within group differences in place versus response strategies at $\mathrm{P}_{\text {crit }}$.

\section{Experiment 2}

All housing, general surgery, training, drug infusion, and statistical procedures were as in Experiment 1. For cannulae implants into the dorsolateral striatum, the stereotaxic coordinates were as follows: nosebar, -3.3 ; AP, -0.3 ; $\mathrm{ML}, \pm 3.8$; and $\mathrm{DV},-2.6 \mathrm{~mm}$ from dura (Paxinos and Watson 1986). The experimental design included four groups: unoperated $(\mathrm{N}=10$; same rats as experiment 1$)$, dorsolateral striatum no injection $(\mathrm{N}=6)$, dorsolateral striatum aCSF $(\mathrm{N}=7)$, and dorsolateral striatum glucose $(\mathrm{N}=9)$. Training of rats in both experiments overlapped in time. In particular, unoperated controls were included at the time of testing the effects of both hippocampal and striatal manipulation and were pooled for statistical comparisons.

Figure 9 illustrates a representative example of cannulae placement in the dorsolateral striatum.

\section{Acknowledgments}

Supported by NIA (AG 07648), NINDS (NS 32914), USDA (0035200-9839), NIDA (DA 016951), and the Alzheimer's Association.

\section{References}

Benton, D., Ruffin, M.P., Lassel, T., Nabb, S., Messaoudi, M., Vinoy, S., Desor, D., and Lang, V. 2003. The delivery rate of dietary carbohydrates affects cognitive performance in both rats and humans. Psychopharmacology 166: 86-90.

Cassel, J.C., Cassel, S., Galani, R., Kelche, C., Will, B., and Jarrard, L. 1998. Fimbria-fornix vs. selective hippocampal lesions in rats: Effects on locomotor activity and spatial learning and memory. Neurobiol. Learn. Mem. 69: 22-45.

Chang, Q. and Gold, P.E. 2003a. Switching memory systems during learning: Changes in patterns of brain acetylcholine release in the hippocampus and striatum in rats. J. Neurosci. 23: 3001-3005.

. 2003b. Intra-hippocampal lidocaine injections impair acquisition of a place task and facilitate acquisition of a response task in rats. Behav. Br. Res. 144: 19-24.

Cook, D. and Kesner, R.P. 1988. Dorsolateral striatum nucleus and memory for egocentric localization. Behav. Neur. Biol. 49: 332-343.

Degroot, A., Kornecook, T., Quirion, R., DeBow, S., and Parent, M.B. 2003. Glucose increases hippocampal extracellular acetylcholine levels upon activation of septal GABA receptors. Br. Res. 979: 71-77.

Denti, A., McGaugh, J.L., Landfield, P.W., and Shinkman, P.G. 1970. Effects of posttrial electrical stimulation on the mesencephalic reticular formation on avoidance learning in rats. Physiol. Behav. 5: 659-662.

Durkin, T.P., Messier, C., de Boer, P., and Westerink, B.H. 1992. Raised glucose levels enhance scopolamine-induced acetylcholine overflow from the hippocampus: An in vivo microdialysis study in the rat. 
Behav. Br. Res. 49: 181-188.

Eichenbaum, H. and Cohen, N.J. 2001. Dissociating multiple memory systems in the brain. In Conditioning to conscious recollection (eds. N.J. Mackintosh et al.), pp. 371-393. Oxford, UK.

Ferbinteanu, J. and McDonald, R.J. 2001. Dorsal/ventral hippocampus, fornix, and conditioned place preference. Hippocampus 11: 187-200.

Gold, P.E. 1986. Glucose modulation of memory storage processing. Behav. Neural Biol. 45: 342-349.

. 1995. The role of glucose in regulating brain and cognition. Am. J. Clin. Nutr. 61: S987-S995.

. 2001. Drug enhancement of memory in aged rodents and humans. In Animal research and human health: Advancing human welfare through behavioral science (eds. M.E. Carroll and J.B.

Overmier), pp. 293-304. American Psychological Association, Washington, DC.

. 2003. Acetylcholine modulation of neural systems involved in learning and memory. Neurobiol. Learn. Mem. 80: 194-210. . 2004. Coordination of multiple memory systems. Neurobiol. Learn. Mem. 82: 230-242.

Gold, P.E., Rose, R.P., and Hankins, L.L. 1978. Retention impairment produced by unilateral amygdala implantation: Reduction by posttrial amygdala stimulation. Behav. Biol. 22: 515-523.

Guillou, J.L., Micheau, J., and Jaffard, R. 1999. Intrahippocampal injections of cysteamine improve the retention of a bar-pressing task in mice. Behav. Br. Res. 103: 113-117.

Hughes, R.N. 2003. Effects of glucose on responsiveness to change in young adult and middle-aged rats. Physiol. Behav. 78: 529-534.

Iaria, G., Petrides, M., Dagher, A., Pike, B., and Bohbot, V.D. 2003. Cognitive strategies dependent on the hippocampus and caudate nucleus in human navigation: Variability and change with practice. J. Neurosci. 23: 5945-5952.

Kesner, R.P., Bolland, B.L., and Dakis, M. 1993. Memory for spatial locations, motor responses, and objects: Triple dissociation among the hippocampus, caudate nucleus, and extrastriate visual cortex. Exp. Brain Res. 93: 462-470.

Knowlton, B.J., Mangels, J.A., and Squire, L.R. 1996. A neostriatal habit learning system in humans. Science 273: 1399-1402.

Kopf, S.R. and Baratti, C.M. 1996. Memory modulation by post-training glucose or insulin remains evident at long retention intervals. Neurobiol. Learn. Mem. 65: 189-191.

Korol, D.L. 2002. Enhancing cognitive function across the life span. Ann. N.Y. Acad. Sci. 959: 167-179.

. 2004. Role of estrogen in balancing contributions from multiple memory systems. Neurobiol. Learn. Mem. 82: 309-323.

Korol, D.L. and Gold, P.E. 1998. Glucose, memory, and aging. Am. J. Clin. Nutr. 67: 764S-771S

Korol, D.L., Malin, E.L., Borden, K.A., Busby, R.A., and Couper-Leo, J. 2004. Shifts in preferred learning strategy across the estrous cycle in female rats. Horm. Behav. 45: 330-338.

Lee, M.H. and Ma, Y.L. 1995. Amphetamine enhances memory retention and facilitates norepinephrine release from the hippocampus in rats. Brain. Res. Bull. 37: 411-416.

Luft, T., Pereira, G.S., Cammarota, M., and Izquierdo, I. 2004. Different time course for the memory facilitating effect of bicuculline in hippocampus, entorhinal cortex, and posterior parietal cortex of rats. Neurobiol. Learn. Mem. 82: 52-56.

Marta-Barros, D., Ramirez, M.R., Dos Reis, E.A., and Izquierdo, I. 2004. Participation of hippocampal nicotinic receptors in acquisition, consolidation and retrieval of memory for one trial inhibitory avoidance in rats. Neuroscience 126: 651-656.

Matthews, D.B., Ilgen, M., White, A.M., and Best, P.J. 1999. Acute ethanol administration impairs spatial performance while facilitating nonspatial performance in rats. Neurobiol. Learn. Mem. 72: 169-179.

McDonald, R.J. and White, N.M. 1993. A triple dissociation of memory systems: Hippocampus, amygdala, and dorsal striatum. Behav. Neurosci. 107: 3-22.

. 1995. Information acquired by the hippocampus interferes with acquisition of the amygdala-based conditioned-cue preference in the rat. Hippocampus 5: 189-197.

McElroy, M.W. and Korol, D.L. 2005. Intrahippocampal muscimol shifts learning strategy in gonadally intact young adult female rats. Learn. Mem. 12: $150-158$.

McIntyre, C.K., Pal, S.N., Marriott, L.K., and Gold, P.E. 2002. Competition between memory systems: Acetylcholine release in the hippocampus correlates negatively with good performance on an amygdala-dependent task. J. Neurosci. 22: 1171-1176.

McIntyre, C.K., Marriott, L.K., and Gold, P.E. 2003. Patterns of brain acetylcholine release predict individual differences in preferred learning strategies in rats. Neurobiol. Learn. Mem. 79: 177-183.

McNay, E.C. and Gold, P.E. 1998. Memory modulation across neural systems: Intra-amygdala glucose reverses deficits caused by intraseptal morphine on a spatial task but not on an aversive task. J.
Neurosci. 18: 3853-3858.

2002. Food for thought: Fluctuations in brain extracellular glucose provide insight into the mechanisms of memory modulation. Behav. Cogn. Neurosci. Rev. 1: 264-280.

McNay, E.C. and Sherwin, R.S. 2004. Effect of recurrent hypoglycemia on spatial cognition and cognitive metabolism in normal and diabetic rats. Diabetes 53: 418-425.

McNay, E.C., Fries, T.M., and Gold, P.E. 2000. Decreases in rat extracellular hippocampal glucose concentration associated with cognitive demand during a spatial task. Proc. Natl. Acad. Sci. 97: 2881-2885

McNay, E.C., McCarty, R.C., and Gold, P.E. 2001. Fluctuations in brain glucose concentration during behavioral testing: Dissociations between brain areas and between brain and blood. Neurobiol. Learn. Mem. 75: 325-337.

Messier, C. 1997. Object recognition in mice: Improvement of memory by glucose. Neurobiol. Learn. Mem. 67: 172-175.

. 2004. Glucose improvement of memory: A review. Eur. J. Pharmacol. 490: 33-57.

Messier, C. and Destrade, C. 1988. Improvement of memory for an operant response by post-training glucose in mice. Behav. Br. Res. 31: 185-191.

Messier, C. and White, N.M. 1984. Contingent and non-contingent actions of sucrose and saccharin reinforcers: Effects on taste preference and memory. Physiol. Behav. 32: 195-203.

1987. Memory improvement by glucose, fructose, and two glucose analogs: A possible effect on peripheral glucose transport Behav. Neural. Biol. 48: 104-127.

Micheau, J., Destrade, C., and Soumireu-Mourat, B. 1984 Time-dependent effects of posttraining intrahippocampal injections of corticosterone on retention of appetitive learning tasks in mice. $J$. Pharmacol. 106: 39-46.

Mitchell, J.A. and Hall, G. 1988. Dorsal striatum-putamen lesions in the rat may impair or potentiate maze learning depending upon availability of stimulus cues and relevance of response cues. Q. J. Exp. Psychol. B. 40: 243-258.

Mizumori, S.J., Yeshenko, O., Gill, K.M., and Davis, D.M. 2004. Parallel processing across neural systems: Implications for a multiple memory system hypothesis. Neurobiol. Learn. Mem. 82: 278-298.

Olton, D.S., Becker, J.T., and Handelmann, G.E. 1979. Hippocampus, space, and memory. Behav. Brain Sci. 2: 313-365.

Packard, M.G. 1998. Posttraining estrogen and memory modulation. Horm. Behav. 34: 126-139.

. 1999. Glutamate infused post-training into the hippocampus or caudate-putamen differentially strengthens place and response learning. Proc. Natl. Acad. Sci. 96: 12881-12886.

Packard, M.G. and Knowlton, B.J. 2002. Learning and memory functions of the basal ganglia. Annu. Rev. Neurosci. 25: 563-593.

Packard, M.G. and McGaugh, J.L. 1992. Double dissociation of fornix and dorsal striatum nucleus lesions on acquisition of two water maze tasks: Further evidence for multiple memory systems. Behav. Neurosci. 106: 439-446.

. 1996. Inactivation of hippocampus or caudate nucleus with lidocaine differentially affects expression of place and response learning. Neurobiol. Learn. Mem. 65: 65-72.

Packard, M.G. and White, N.M. 1991. Dissociation of hippocampus and dorsal striatum nucleus memory systems by posttraining intracerebral injection of dopamine agonists. Behav. Neurosci. 105: 295-306.

Packard, M.G., Hirsh, R., and White, N.M. 1989. Differential effects of fornix and caudate nucleus lesions on two radial maze tasks: Evidence for multiple memory systems. J. Neurosci. 9: 1465-1472.

Packard, M.G., Cahill, L., and McGaugh, J.L. 1994. Amygdala modulation of hippocampal-dependent and dorsal striatum nucleus-dependent memory processes. Proc. Natl. Acad. Sci. 91: 8477-8481.

Packard, M.G., Kohlmaier, J.R., and Alexander, G.M. 1996. Posttraining intrahippocampal estradiol injections enhance spatial memory in male rats: Interaction with cholinergic systems. Behav. Neurosci. 110: $626-632$.

Parent, M.B. and Gold, P.E. 1997. Intra-septal infusions of glucose potentiate inhibitory avoidance deficits when co-infused with the GABA agonist muscimol. Brain Res. 745: 317-320.

Parent, M.B., Laurey, P.T., Wilkniss, S., and Gold, P.E. 1997. Intraseptal infusions of muscimol impair spontaneous alternation performance: Infusions of glucose into the hippocampus, but not the medial septum, reverse the deficit. Neurobiol. Learn. Mem. 68: 75-85.

Pavone, F., Capone, F., Battaglia, M., and Sansone, M. 1998. Shuttle-box avoidance learning in mice: Improvement by combined glucose and tacrine. Neurobiol. Learn. Mem. 69: 204-210.

Paxinos, G. and Watson, C. 1986. The rat brain in stereotaxic coordinates. Academic Press, San Diego, CA. 
Poldrack, R.A. and Packard, M.G. 2003. Competition among multiple memory systems: Converging evidence from animal and human brain studies. Neuropsychologia. 41: 245-251.

Poldrack, R.A. and Rodriguez, P. 2004. How do memory systems interact? Evidence from human classification learning. Neurobiol. Learn. Mem. 82: 324-332.

Poldrack, R.A., Clark, J., Paré-Blagoev, J., Shohamy, D., Creso Moyano, J., Myers, C., and Gluck, M.A. 2001. Interactive memory systems in the human brain. Nature 414: 546-550.

Ragozzino, M.E. and Gold, P.E. 1994. Task-dependent effects of intra-amygdala morphine injections: Attenuation by intra-amygdala glucose injections. J. Neurosci. 14: 7478-7485.

. 1995. Glucose injections into the medial septum reverse the effects of intraseptal morphine infusions on hippocampal acetylcholine output and memory. Neuroscience 68: 981-988.

Ragozzino, M.E., Parker, M.E., and Gold, P.E. 1992. Spontaneous alternation and inhibitory avoidance impairments with morphine injections into the medial septum: Attenuation by glucose administration. Brain Res. 597: 241-249.

Ragozzino, M.E., Unick, K.E., and Gold, P.E. 1996. Hippocampal acetylcholine release during memory testing in rats: Augmentation by glucose. Proc. Natl. Acad. Sci. 93: 4693-4698.

Ragozzino, M.E., Pal, S.N., Unick, K., Stefani, M.R., and Gold, P.E. 1998. Modulation of hippocampal acetylcholine release and spontaneous alternation scores by intrahippocampal glucose injections. $J$. Neurosci. 18: 1595-1601.

Rashidy-Pour, A. 2001. ATP-sensitive potassium channels mediate the effects of a peripheral injection of glucose on memory storage in an inhibitory avoidance task. Behav. Br. Res. 126: 43-48.

Restle, E. 1957. Discrimination of cues in mazes: A resolution of the "place-vs.-response" question. Psychol. Rev. 64: 217-228.

Roozendaal, B., Nguyen, B.T., Power, A.E., and McGaugh, J.L. 1999. Basolateral amygdala noradrenergic influence enables enhancement of memory consolidation induced by hippocampal glucocorticoid receptor activation. Proc. Natl. Acad. Sci. 96: 11642-11647.

Sansone, M., Battaglia, M., and Pavone, F. 2000. Shuttle-box avoidance learning in mice: Improvement by glucose combined with stimulant drugs. Neurobiol. Learn. Mem. 73: 94-100.

Schroeder, J.P. and Packard, M.G. 2003. Systemic or intra-amygdala injections of glucose facilitate memory consolidation for extinction of drug-induced conditioned reward. Eur. J. Neurosci. 17: 1482-1488.

Schroeder, J.P., Wingard, J.C., and Packard, M.G. 2002. Post-training reversible inactivation of hippocampus reveals interference between memory systems. Hippocampus 12: 280-284.

Shah, A.A. and Parent, M.B. 2003. Septal infusions of glucose or pyruvate, but not fructose, produce avoidance deficits when co-infused with the GABA agonist muscimol. Neurobiol. Learn. Mem. 79: $243-251$.

Stefani, M.R. and Gold, P.E. 1998. Intra-septal injections of glucose and glibenclamide attenuate galanin-induced spontaneous alternation performance deficits in the rat. Brain Res. 813: 50-56.

. 2001. Intrahippocampal infusions of k-atp channel modulators influence spontaneous alternation performance: Relationships to acetylcholine release in the hippocampus. J. Neurosci. 21: 609-614.

Stefani, M.R., Nicholson, G.M., and Gold, P.E. 1999. ATP-sensitive potassium channel blockade enhances spontaneous alternation performance in the rat: A potential mechanism for glucose-mediated memory enhancement. Neuroscience 93: 557-563.

Talley, C.E.P., Kahn, S., Alexander, L., and Gold, P.E. 2000. Epinephrine fails to enhance performance of food-deprived rats on a delayed spontaneous alternation task. Neurobiol. Learn. Mem. 73: 79-86.

Tolman, E.C., Ritchie, B.F., and Kalish, D. 1946. Studies in spatial learning, II: Place learning versus response learning. J. Exp. Psychol. 35: 221-229.

. 1947. Studies in spatial learning, V: Response versus place learning by the noncorrection method. J. Exp. Psychol. 37: 285-292.

Voermans, N.C., Petersson, K.M., Daudey, L., Weber, B., van Spaendonck, K.P., Kremer, P.H., and Fernandez, G. 2004. Interaction between the human hippocampus and the caudate nucleus during route recognition. Neuron 43: 427-435.

Walker, J.A. and Olton, D.S. 1979. Spatial memory deficit following fimbria-fornix lesions: Independent of time for stimulus processing. Physiol. Behav. 23: 11-15.

Watson, G.S. and Craft, S. 2004. Modulation of memory by insulin and glucose: Neuropsychological observations in Alzheimer's disease. Eur. J. Pharmacol. 490: $97-113$.

White, N.M. and McDonald, R.J. 1993. Acquisition of a spatial conditioned place preference is impaired by amygdala lesions and improved by fornix lesions. Behav. Br. Res. 55: 269-281.

. 2002. Multiple parallel memory systems in the brain of the rat. Neurobiol. Learn. Mem. 77: 125-184.

Received October 22, 2004; accepted in revised form May 6, 2005.
374 Learning \& Memory

www.learnmem.org 


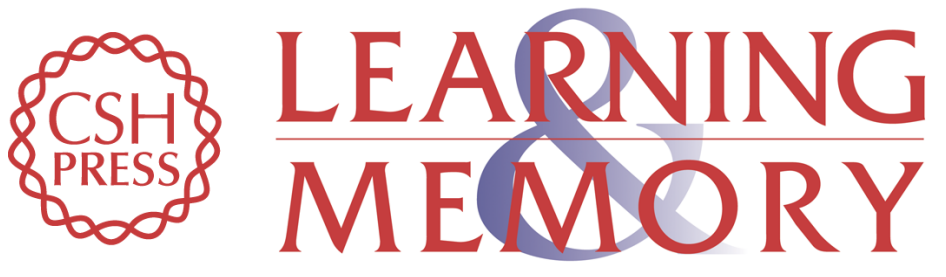

\section{Glucose injections into the dorsal hippocampus or dorsolateral striatum of rats prior to T-maze training: Modulation of learning rates and strategy selection}

Clinton E. Canal, Sonja J. Stutz and Paul E. Gold

Learn. Mem. 2005, 12:

Access the most recent version at doi:10.1101/lm.88205

References This article cites 84 articles, 18 of which can be accessed free at: http://learnmem.cshlp.org/content/12/4/367.full.html\#ref-list-1

License

Email Alerting

Receive free email alerts when new articles cite this article - sign up in the box at the Service top right corner of the article or click here. 